\title{
Évolution de la bande dessinée en France depuis quinze ans
}

\author{
Gilles Ratier \\ Écrivain, journaliste et \\ Secrétaire de l'ACBD
}

\begin{abstract}
Avant l'an 2000
Pratiquement depuis ses origines (à l'exception des albums de Rodolphe Töpffer, le créateur du $9^{\text {ème }}$ art, en 1835), la bande dessinée francophone européenne passait, la plupart du temps, par une prépublication dans les journaux. Or, petit à petit, et particulièrement dans les années 1990, la bande dessinée ne va plus se destiner uniquement à la presse et va devenir un média à part entière. L'album a pris le dessus, profitant de cette mutation. Cependant, ce nouveau support, en s'adressant de plus en plus à un public adulte, se coupe alors d'une partie du lectorat populaire, à l'exception des immuables Astérix, Lucky Luke, Blake et Mortimer, et autres Schtroumpfs. À la fin des années 1990, rares étaient les créations, datant de moins de 20 ans, qui vendaient à plus de 100000 exemplaires. C'était pourtant le cas du Petit Spirou, de XIII, de Largo Winch, de Titeuf (qui n'en était alors qu'à ses débuts) ou d'albums d'auteurs comme Enki Bilal, Jacques Tardi, André Juillard ou François Bourgeon ; leur succès s'expliquant par une maitrise de la narration et du dessin. Les éditeurs prenaient alors doucement conscience de l'importance du scénario (lesquels commençaient à exploiter des thèmes plus sociaux et politiques, à l'instar de ceux écrits par Pierre Christin, Didier Convard ou Frank Giroud) alors que les mangas (c'est-à-dire les bandes dessinées japonaises) commençaient, timidement, à avoir les faveurs d'un public peu aisé, ayant du mal à débourser le prix d'un album cartonné.

Pour bien comprendre l'évolution de la BD entre 1990 et 1999, il faut savoir que les cartes éditoriales ne se répartissaient plus qu'entre quelques grosses maisons et, qu'à quelques exceptions près, on retrouve encore les mêmes de nos jours! Ces dernières avaient bâti leur succès économique et leur catalogue avec des revues qui ont pratiquement toutes disparues aujourd'hui: ces magazines étaient, principalement, des périodiques de divertissement, ce qui explique le fait que les composantes de la bande dessinée se résumaient alors à l'humour et à l'aventure. Á l'époque, l'ordre d'importance économique de ces maisons d'éditions était le suivant : les éditions Dupuis (dont le catalogue était axé sur le contenu de l'hebdomadaire Spirou, avec des grands dessinateurs belges comme Jijé, Peyo, Franquin et Morris), les éditions Dargaud et Le Lombard (les
\end{abstract}


premières bâties sur le magazine Pilote avec des auteurs aussi importants qu'Uderzo et Goscinny ou Charlier, Giraud/Moebius, Gottlieb, Fred, Mézières et Christin, Druillet, Bretécher, etc. ; le second publiant la plupart des auteurs du journal Tintin comme Jacobs, Cuvelier, Hermann, Vance, etc., les héros « ligne claire » d'Hergé et de Jacques Martin, publiés dans cet hebdomadaire, étant exploités en albums par les éditions Casterman), les éditions Casterman et A.U.D.I.E. (le premier avec le mensuel (A Suivre) qui réunissait Forest, Pratt, Tardi, Schuiten et Peeters..., et le second avec le mensuel d'humour Fluide Glacial qui révèlera Binet, Tronchet, Maëster, Coyote...).

Puis, on trouvait aussi des éditeurs plus récents comme le groupe Glénat qui a intégré les éditions Vents d'Ouest (et qui publiait les magazines Circus et Vécu), Les Humanoïdes associés (avec Métal Hurlant), et Albin Michel (avec L'Écho des Savanes où se retrouvaient certains auteurs de Charlie-Hebdo ou d'Hara-Kiri comme Reiser, Wolinski, Pétillon et Vuillemin), sans oublier l'omniprésent groupe Hachette avec Disney Presse, le $\mathrm{n}^{\circ} 1$ du réseau presse (avec Le Journal de Mickey et Picson Magazine), lequel, cependant, n'investissait cependant absolument pas le secteur album.

Notons que les magazines L'Écho des Savanes, Métal Hurlant ou Fluide Glacial ont été créés, à l'origine, par des dessinateurs ou des scénaristes issus de l'hebdomadaire Pilote. Ces différentes grandes structures étaient suivies, dans une moindre mesure, par Delcourt et Soleil; éditeurs qui vont devenir, petit à petit, beaucoup plus importants. Cependant, tous ces acteurs vivaient sur leurs acquis et étaient à la recherche du moindre bestseller. Les auteurs ayant déjà fait leurs preuves étaient, bien entendu, choyés ; surtout quand certains, comme Jean Van Hamme (le scénariste de XIII, Thorgal et autres Largo Winch) arrivaient à faire vendre des albums rien que sur leur simple nom. Pendant ce temps-là, une nouvelle génération d'auteurs, issue des fanzines ou des petites structures, s'essayait à d'autres expériences graphiques et narratives en s'inspirant de la littérature et de la peinture, et commençait enfin à percer auprès du grand public : la production bande dessinée allait alors bénéficier d'un renouvellement de la création et d'une grande diversification de son lectorat !

\section{Depuis l'an 2000}

\section{1) Une énorme production}

En l'an 2000, 1563 livres appartenant au monde de la bande dessinée avaient été publiés : une évolution à la hausse constatée depuis déjà 5 années. En effet, au début des années 1990, on atteignait à peine le chiffre de 800 parutions! Ce n'était pourtant qu'un début puisqu'en 2012 nous sommes arrivés au chiffre faramineux de 5565 livres concernant 
l'univers de la bande dessinée ; alors que cette inflation diminue la visibilité d'au moins 6 livres sur 10 en librairie. Outre la forte poussée des bandes dessinées asiatiques dont nous parleront plus en détail par la suite, il faut signaler la hausse du nombre de titres édités par les éditeurs traditionnels et par les labels indépendants (ou alternatifs), lesquels privilégient de nouvelles voies alors qu'ils sont de plus en plus noyés dans la masse. En comparaison, 65500 livres écrits en français (tous genres confondus) ont été publiés en 2012 ; la bande dessinée représenterait donc 7,38\% des livres édités sur le territoire francophone et un peu plus de 6,5\% du chiffre d'affaires de l'édition. Malgré la crise ambiante, l'industrie du livre a choisi la diversité de sa production et la bande dessinée (expression culturelle très bien segmentée, qui reste l'un des plus dynamiques secteurs du marché du livre) montre l'exemple.

Alors que la profession s'interroge sur la surabondance de l'offre, il semble que cette diversité, bien orchestrée, soit, à l'inverse des craintes, bénéfique à un marché qui n'est pourtant pas indéfiniment extensible : il est estimé à 250 millions d'euros, soit un peu plus de 30 millions d'albums vendus par an, soit 1 livre sur 7 vendus en France. Mais attention, les acheteurs concentrent leurs achats sur les valeurs sûres et les libraires, submergés, réalisent des mises en place de plus en plus faibles sur les albums peu rentables. Enfin, notons que c'est surtout le dernier trimestre de l'année qui permet de donner un nouveau souffle aux ventes; période où les éditeurs font le plus gros de leur chiffre d'affaires : avec (ou malgré) la parution de $60 \%$ des principaux «blockbusters» de l'année et pratiquement $40 \%$ de la production annuelle.

2) De plus en plus d'éditeurs (326 en 2012), alors que seulement une poignée d'entre eux (4 groupes leaders en 2012, suivis par 12 autres entreprises) publie la quasi-totalité des albums de BD

En effet, c'est toujours le même cercle très fermé d'éditeurs qui produit le plus. Le groupe Média-Participations (c'est-à-dire Dargaud, Le Lombard, Kana, Dupuis et Urban Comics) contrôle 27\% du marché de la bande dessinée francophone, et a publié, en 2012, 14\% de la production bande dessinée. On retrouve, dans le groupe Média-Participations, les principales vedettes de la bande dessinée d'aujourd'hui : Le Petit Spirou, Lucky Luke, Blake et Mortimer, XIII, Largo Winch, Cédric, Boule et Bill, Thorgal...

Les éditions Delcourt sont devenues le groupe le plus prolifique du marché $\left(16 \%\right.$ de la production) et arrive en $2^{\text {ème }}$ position en chiffre d'affaires $(16,3 \%)$. Si le catalogue de la maison mère se composait, à l'origine, principalement de séries fantastique, il s'est énormément diversifié depuis, allant des Blagues de Toto et des Légendaires aux ouvrages esthétisants 
de Chris Ware ou aux reportages dessinés de Guy Delisle, en passant par le comics Walking Dead ou par les mangas des labels Akata et Tonkam. Guy Delcourt a aussi racheté, en 2011, le groupe Soleil spécialisé dans l'beroicfantasy (son principal best-seller, Lanfeust, ayant même donné son nom à un magazine).

Le groupe Glénat (avec sa propre marque et les labels Glénat Mangas et Vents d'Ouest) demeure en $3^{\text {ème }}$ position, tant au niveau de la production avec $8,6 \%$ qu'en chiffres d'affaires avec $16,2 \%$, surtout grâce aux mangas (Dragon Ball et One Piece) et aux séries Titeuf et Lou. Il a racheté le département bande dessinée d'Albin Michel qui publiait les auteurs issus du mensuel L'Écho des Savanes qu'il a également ressuscité, et s'est associé avec Disney Presse pour diffuser en librairie les albums avec Mickey, Donald, Picsou, etc.

Le groupe Gallimard qui avait déjà le label Futuropolis et qui a racheté récemment le groupe Flammarion (avec Casterman, Jungle! et Fluide Glacial), est le $4^{\text {eme }}$ en production avec $6 \%$ et en chiffres d'affaires avec 9,6\%, surtout grâce à l'exploitation des albums de Tintin, du Chat de Geluck, des Simpson en BD ou de ceux d'auteurs célèbres comme Tardi, Bilal et Loisel.

Viennent ensuite d'autres maisons très productives comme les comics et mangas de Panini, les filiales BD du géant de l'édition francophone Hachette (avec les mangas de Pika ou Albert-René qui n'a qu'une seule série à son catalogue, mais quelle série puisqu'il s'agit d'Astérix !), quelques éditeurs de mangas comme Kazé Manga (qui appartient à deux des plus importants éditeurs de mangas japonais), Kurokawa (qui, lui, est une filiale du groupe Editis), Taïfu et Ki-oon, ainsi que des maisons plus classiques comme Bamboo, Clair de Lune, Les Humanoïdes associés, Paquet, EP, Bayard et Marsu.

Citons aussi les récentes maisons indépendantes que sont 12 bis (avec Les Passagers du vent) ou Mad Fabrik (avec Kid Paddle) qui ont été créées par des dissidents des grandes structures en place. Quoi qu'il en soit, ces ténors du secteur publient plus de $72 \%$ de la production en titres et réalisent, à eux seuls, plus des $2 / 3$ des activités de la bande dessinée francophone.

Ce qui veut dire que ces prolifiques éditeurs laissent peu de marge de manœuvre aux labels alternatifs (L'Association, La Boîte à Bulles, Ça et Là, Cornélius, Ego comme X, Les Enfants Rouges, Mosquito, Rackham, Les Requins Marteaux, 6 Pieds sous terre, Vertige Graphic) ou aux nouveaux venus, éditeurs généralistes et littéraires comme Actes Sud, Sarbacane, Olivius, La Martinière, L'École des loisirs et L'Harmattan; à noter que ces derniers sont cependant bien armés sur le plan diffusion et 
distribution et qu'ils n'hésitent pas à chasser sur les terres des petites structures, lesquelles doivent avoir, aujourd'hui, un catalogue très pertinent pour être viable. Par ailleurs, la visibilité en librairies est d'autant plus difficile à assurer que la filière du livre est en pleine mutation : les achats sur Internet, par exemple, ne cessant d'augmenter!

\section{3) Implantation des mangas (BD japonaises) et des comics (BD américaines tendance super-héros)}

Toutes ces bandes dessinées, de différentes origines ou appartenant à un genre bien défini, touchent des publics importants mais souvent différents de ceux qui apprécient la traditionnelle bande dessinée francobelge. Devant l'importance prise par la BD japonaise ou américaine, les éditeurs francophones ont tissé des liens suscitant une collaboration entre des artistes venus d'horizons différents : le marché francophone étant l'un des plus ouverts au monde extérieur. Désormais, nous assistons à de véritables échanges : certains auteurs arrivent à travailler aux USA, des cadors de la BD franco-belge planchent sur des versions exportables des plus célèbres super-héros, des projets similaires concernant les mangas sont en cours, et de nombreux auteurs francophones et asiatiques n'hésitent plus à travailler ensemble. D'ailleurs, de plus en plus d'auteurs s'inspirent des codes graphiques et narratifs des mangas ou des comics, comme le prouve nombre de séries publiées par les principaux éditeurs. Et le catalogue de ces derniers rassemble de plus en plus de graphistes, de diverses origines, maittrisant un trait où l'on sent cette nette influence : iraiton alors vers un style universel ? C'est en effet la question que l'on peut se poser!

C'est la bande dessinée asiatique qui est, aujourd'hui, la plus traduite sur le territoire francophone: en 2012, les mangas japonais, manhwas coréens et manhuas chinois étaient au nombre de 1586 (38,6\% des nouveautés de l'année) alors qu'en 1994, seulement 19 mangas avaient été traduits en français. Le phénomène ne se limite pas à nos frontières puisque, actuellement, c'est toute l'Europe et même les États Unis qui se sont laissé séduire par les bandes dessinées asiatiques. Grâce aux mangas, la bande dessinée a retrouvé un public populaire (plus jeune et plus féminin), sensible au fait que ces ouvrages soient peu onéreux (pour une quantité importante de pages) et que les nouveaux tomes de chaque série se succèdent dans des délais très rapprochés : c'est d'ailleurs un facteur non négligeable pour l'augmentation de la production bande dessinée.

Aujourd'hui, toutes les librairies ont des rayons spécialisés en mangas et tous les éditeurs traditionnels de bande dessinée ont une collection ou un label spécialisé dans ce secteur très concurrentiel. Les mangas japonais, manhwas coréens, manhuas chinois et assimilés (c'est-àdire des bandes dessinées qui ont le goût du manga mais qui sont réalisées 
par des auteurs européens) représenteraient environ 30\% du marché de la bande dessinée francophone et donc l'achat d'1 album sur 3 .

Les 10 plus grosses vedettes des mangas, publiées chez 5 éditeurs, seulement, qui assurent plus de la moitié des ventes dans leur globalité sont Naruto (chaque volume étant tiré à 225000 exemplaires), One Piece (165 000 exemplaires par tome au rythme de 6 par an), Fairy Tail, Black Butler, Bleach, Bakuman, Hunter X Hunter, Judge, Pokemon, Soul Eater, sans oublier les rééditions de Dragon Ball, de Fullmetal Alchemist, de Death Note, de Fruits Basket et de Nana, qui continuent à se vendre régulièrement. Longtemps décriée, la bande dessinée japonaise a pourtant trouvé sa place dans le paysage culturel. Pour preuve : le succès du festival «Japan Expo » au Parc des expositions de Paris-Nord Villepinte (208 000 visiteurs en 3 jours, et la mise en avant de nombreuses récompenses éditoriales dont le prix Asie des critiques et journalistes de bandes dessinées : le $1^{\text {er }}$ ayant été décerné à Gen d'Hiroshima de Keiji Nakazawa, aux éditions Vertige Graphic).

Si le nombre de magazines publiant des séries américaines superhéroïques (Spider-Man, X-Men, Superman, Batman, etc.,) se stabilise, il y a de plus en plus de comics qui sont publiés en albums: en 2012, ils représentaient 10,9\% des nouvelles publications de bandes dessinées. Ce secteur qui décline pourtant, chaque année, dans son pays d'origine est, heureusement pour lui, porté par les nombreuses adaptations cinématographiques à succès du moment: outre les séries déjà citées, signalons aussi The Avengers, Iron Man, Hulk et Fantastic Four. En France, la bande dessinée de super-héros est dominée par le groupe Panini France qui a l'exclusivité des BD Marvel comme X-Men ou Spiderman et, tout récemment, par la filiale Urban Comics de Média-Participations qui a récupéré les droits d'édition de l'éditeur américain DC (Batman et Superman).

\section{4) Succès des valeurs sûres et émergence des romans graphiques}

Alors que le tirage moyen baisse toujours, la bande dessinée reste toutefois à l'origine de gros succès dans l'édition francophone, tous genres confondus. Par exemple, jamais un livre n'aura été tiré à autant d'exemplaires que le dernier Astérix (3 178000 exemplaires) ! Mais ce n'est pas le seul : Titeuf, par exemple, est un véritable phénomène de société (ses albums sont tirés à 1000000 exemplaires). Par ailleurs, la reprise de Lucky Luke (450 000 exemplaires) ou celle de Blake et Mortimer (440 000 exemplaires) sont aussi d'énormes succès, tout comme chaque nouvel album de Largo Winch et de XIII (respectivement 440000 exemplaires et 350 000, chacun), ou encore de Boule et Bill (300 000 exemplaires), Le Chat, Kid Paddle et Lou (250 000 exemplaires).

Notons également le succès obtenu par des séries au concept original comme Le Décalogue ou Le Triangle secret (120 000 exemplaires par 
titres), conçues autour du travail d'un scénariste. Même des bandes dessinées plus personnelles, réalisées par des auteurs célèbres, comme Tardi, Bilal, Loisel, Bourgeon, Pétillon, Schuiten et Peeters (entre 100000 et 200000 exemplaires par titres), se hissent aussi sur les rangs des meilleures ventes.

C'est également le cas pour certains jeunes talents comme Joann Sfar et son Chat du rabbin, Manu Larcenet et son Blast, Lewis Trondheim et son Lapinot, Emmanuel Guibert et son Photographe ou Marjane Satrapi et son Persépolis (albums tirés autour de 100000 exemplaires) : c'est une nouvelle génération d'auteurs prolifiques (on pourrait aussi citer Étienne Davodeau, Pascal Rabaté, Marc-Antoine Mathieu ou David B.), réalisant indifféremment textes ou dessins, et qui continuent à proposer des bandes dessinées plus expérimentales chez les éditeurs indépendants.

Ce mouvement défend une bande dessinée d'“auteur"; notons qu’il avait été amorcé, dans les années 1990, par des personnalités comme Edmond Baudoin, Baru, Dupuy et Berberian, Jean-C. Denis, Farid Boudjellal ou Jean-Philippe Stassen, qui ont été les premiers, en langue française, à réaliser ce que l'on appelle désormais des romans graphiques, à l'instar de ceux avaient été réalisés aux USA par Will Eisner ou Art Spiegelman. C'est-à-dire une bande dessinée qui ressemble, autant par sa forme que par son contenu, à un roman : plus petit format, plus grande pagination, principalement en noir et blanc, plus grande liberté graphique et narrative, etc.

\section{5) Confirmation de l'album comme support principal de la BD}

Selon les résultats d'une enquête du ministère de la Culture et de la Communication sur les pratiques culturelles des Français à l'ère numérique, on n'aurait jamais autant lu qu'aujourd'hui... Mais l'ordinateur s'est substitué aux supports de connaissances et de loisirs traditionnels, au point que les sociologues parlent désormais de «culture d'écran», ceci au détriment de la lecture sur papier et, particulièrement, de celle de la presse : d'où la nécessité, pour les éditeurs de magazines de prolonger leur lectorat sur le numérique et Internet !

Alors que la presse fut très longtemps le support privilégié de publication pour la bande dessinée, c'est aujourd'hui l'album (qui n'est plus obligatoirement cartonné et qui se présente sous des formats de plus en plus divers) qui a pris le dessus. D'ailleurs, même s'il y a de plus en plus de magazines généralistes qui proposent des bandes dessinées, la presse de bande dessinée souffre énormément de la concurrence de l'album : et il n'y a plus que 16 véritables revues de bandes dessinées distribuées en kiosques qui proposent des créations de bandes dessinées européennes : Spirou, Le 
Journal de Mickey, Picsou Magazine, Tchô, Lanfenst Mag ou Fluide Glacial pour les plus connues...

En revanche, l'intérêt pour la bande dessinée est de plus en plus vivace même si les revues qui parlent du $9^{\mathrm{e}}$ art comme CaseMate ou les $[d B D]$ ont du mal à être viables en kiosques. Par ailleurs, d'autres magazines sont totalement gratuits (comme Zoo) tandis que d'autres se vendent en librairies ou uniquement par correspondance, comme la référence historique qu'est Hop! ou encore L'Avis des Bulles pour les bibliothécaires.

Cependant, l'actualité de la bande dessinée se retrouve surtout sur les sites informatifs et non commerciaux d'Internet, lesquels sont de plus en plus performants et consultés. Parmi eux, citons actuabd.com, bdroom.com, bodoi.com, bdgest.com, auracan.com, du9.org, planetebd.com et sceneario.com (dont les fréquentations moyennes avoisinent les 100000 visiteurs uniques par mois). Le Net, avec ses "chats", blogs (dont certains jeunes auteurs comme Boulet ou Pénélope Bagieu ont émergé et ont finalement trouvé un méritoire succès public chez les éditeurs en place), forums, bandes dessinées inédites, achats en ligne mais aussi, hélas, ses piratages, est devenu, aujourd'hui, le lieu où la bédéphilie s'exprime le plus.

Il faut bien aussi reconnaitre qu'aujourd'hui, les moteurs qui tirent la consommation sont plus que jamais le cinéma, la télévision, les dessins animés, les jeux vidéo et toutes les applications de l’industrie numérique. La commercialisation du 9e art est en train de changer: ne serait-ce que par l'omniprésence de ces nouvelles technologies qui favorisent une visibilité des stocks que ne permet plus la librairie et poussent, quelquefois malgré eux, les différents acteurs de la chaîne du livre à s'adapter !

L'édition numérique est donc l'une de ces évolutions non négligeables que les acteurs du secteur prennent en compte sérieusement. Mais nous n'en sommes qu'à un stade balbutiant et nous ne pouvons pas encore juger de l'importance du chiffre d'affaires de cette forme virtuelle de l'édition; même si l'on sait qu'il est actuellement minuscule: les acteurs les plus avisés estiment, qu'aujourd'hui, il n'y aurait que $0,1 \%$ des livres (tous genres confondus) qui se vendraient en fichier numérique, en Europe francophone. On ne sait pas ce qu'il en sera demain, mais ce qui est sûr, c'est que les éditeurs se préparent à un éventuel virage numérique, ne tenant pas à réitérer les erreurs de l'industrie musicale et cinématographique. D'ailleurs, l'offre commence aussi à se structurer: quelques sociétés se disputant les « best-sellers » et les auteurs se mobilisant sur le sujet.

\section{6) Concentration sur les autres medias}

Source d'inspiration pour d'autres formes d'art et de supports, la 
bande dessinée prend de plus en plus conscience de sa valeur. L'intérêt exponentiel des milieux audiovisuels est un facteur supplémentaire qui favorise sa légitimation. La bande dessinée est devenue un fantastique vivier de scénarios car l'évolution des technologies en facilite l'adaptation. On retrouve ce même engouement pour la bande dessinée dans les dessins animés, dans les jeux vidéo ou de société, dans les pièces de théâtre, dans le domaine musical et, bien sûr, dans les nouvelles technologies. Quant aux produits dérivés, leur exploitation prend une véritable tournure industrielle; et n'oublions pas le secteur publicitaire qui est de plus en plus friand d'images BD. L'exemple le plus frappant est celui de l'utilisation, il y a quelques années maintenant, de Largo Winch et de Corto Maltese pour les parfums Dior: ces deux visuels ont touché plus gens que les précédentes campagnes réalisées avec Johnny Hallyday et Zinédine Zidane!

Manifestement, La bande dessinée est descendue dans la rue et redynamise l'image de toutes sortes de produits car c'est devenu un art graphique et littéraire qui s'expose et voyage facilement; toutefois, il faut signaler que son expansion s'est construite sans réelle vitrine médiatique. Ainsi, en dehors de l'événementiel Festival d'Angoulême (qui représente, et on peut le regretter, $90 \%$ de la couverture journalistique du domaine), la population s'estime toujours aussi mal informée. En fait, c'est surtout à la télévision (qui a encore du mal à admettre la bande dessinée comme une culture respectable), que le bât blesse. Á l'exception de notables émissions sur Arte ou sur France 2, l'intérêt porté par le petit écran au $9^{\mathrm{e}}$ art est bien moindre que celui des autres médias, alors que la bande dessinée est de plus en plus enracinée dans les foyers et dans les pratiques culturelles.

En effet, aujourd'hui, $30 \%$ des Français se déclarent être intéressés par la bande dessinée : un chiffre révélateur de sa légitimité auprès d'une population qui la considère aussi comme éducative, originale et populaire. 
Alternative Francophone vol.1, 6(2013): 80-89

http://ejournals.library.ualberta.ca/index.php/af

\section{Bibliographie}

Ratier, F. (2013). Une année de bandes dessinées sur le territoire francophone européen, site de l'Association des Critiques et Journalistes de Bande Dessinée), http://www.acbd.fr/category/les-bilans-de-l-acbd/, consulté le 11 janvier 2014, 33 p. 